\title{
Surveillance
}

\section{for sulfadoxine-pyrimethamine resistant malaria parasites in the Lake and Southern Zones, Tanzania, using pooling and next-generation sequencing}

Jeremiah M. Ngondi ${ }^{*}$, Deus S. Ishengoma², Stephanie M. Doctor ${ }^{3}$, Kyaw L. Thwai ${ }^{3}$, Corinna Keeler ${ }^{3}$, Sigsbert Mkude ${ }^{4}$, Oresto M. Munishi ${ }^{1}$, Ritha A. Willilo ${ }^{1}$, Shabbir Lalji ${ }^{1}$, Naomi Kaspar ${ }^{5}$, Chonge Kitojo ${ }^{5}$, Lynn A. Paxton', Nicholas J. Hathaway', Jeffrey A. Bailey ${ }^{7}$, Jonathan J. Juliano ${ }^{3}$, Steven R. Meshnick ${ }^{3}$ and Julie Gutman ${ }^{8 *}$

\begin{abstract}
Background: Malaria in pregnancy (MiP) remains a major public health challenge in areas of high malaria transmission. Intermittent preventive treatment in pregnancy (IPTp) with sulfadoxine-pyrimethamine (SP) is recommended to prevent the adverse consequences of MiP. The effectiveness of SP for IPTp may be reduced in areas where the dhps581 mutation (a key marker of high level SP resistance) is found; this mutation was previously reported to be common in the Tanga Region of northern Tanzania, but there are limited data from other areas. The frequency of molecular markers of SP resistance was investigated in malaria parasites from febrile patients at health centres (HC) in seven regions comprising the Lake and Southern Zones of mainland Tanzania as part of the ongoing efforts to generate national-wide data of SP resistance.
\end{abstract}

Methods: A cross-sectional survey was conducted in the outpatient departments of $14 \mathrm{HCs}$ in seven regions from April to June, 2015. 1750 dried blood spot (DBS) samples were collected (117 to 160 per facility) from consenting patients with positive rapid diagnostic tests for malaria, and no recent (within past 2 months) exposure to SP or related drugs. DNA was extracted from the DBS, pooled by HC, and underwent pooled targeted amplicon deep sequencing to yield estimates of mutated parasite allele frequency at each locus of interest.

Results: The dhps540 mutation was common across all 14 sites, ranging from 55 to $98.4 \%$ of sequences obtained. Frequency of the dhps581 mutation ranged from 0 to 2.4\%, except at Kayanga HC (Kagera Region, Lake Zone) where $24.9 \%$ of sequences obtained were mutated. The dhfr 164 mutation was detected only at Kanyanga HC (0.06\%).

Conclusion: By pooling DNA extracts, the allele frequency of mutations in 14 sites could be directly determined on a single deep-sequencing run. The dhps540 mutant was very common at all locations. Surprisingly, the dhps581 was common at one health center, but rare in all the others, suggesting that there is geographic micro-heterogeneity in mutant distribution and that accurate surveillance requires inclusion of multiple sites. A better understanding of the effect of the dhps581 mutant on the efficacy of IPTP-SP is needed.

\footnotetext{
*Correspondence: jngondi@rti.org; fff2@cdc.gov

${ }^{1}$ RTI International, Dar es Salaam, Tanzania

${ }^{8}$ Malaria Branch, Division of Parasitic Diseases and Malaria, US Centers

for Disease Control and Prevention, Atlanta, GA, USA

Full list of author information is available at the end of the article
} 
Keywords: Plasmodium falciparum, Sulfadoxine-pyrimethamine, Tanzania, Malaria, Resistance

\section{Background}

Malaria in pregnancy $(\mathrm{MiP})$ remains a major public health challenge in areas of high malaria transmission. In pregnant women, malaria can cause mild to severe maternal anaemia, particularly in primigravid women, and placental infection can interfere with the maternalfetal exchange of nutrients and oxygen, leading to preterm delivery and low birth weight, and consequently increasing neonatal mortality $[1,2]$. In order to prevent the adverse consequences of MiP, the World Health Organization (WHO) recommends the use of intermittent preventative treatment in pregnancy (IPTp) with sulfadoxine-pyrimethamine (SP) - a full treatment dose administered to pregnant women during routine ANC visits in the 2nd and 3rd trimesters. IPTp has a protective efficacy of nearly $25 \%$ against low birth weight and $21 \%$ against neonatal mortality [3].

Plasmodium falciparum resistance to SP results from an ordered accumulation of mutations in two genes, namely $P$. falciparum dihydrofolate reductase (Pfdhfr) and $P$. falciparum dihydropteroate synthase (Pfdhps) that code for enzymes targeted by sulfadoxine and pyrimethamine, respectively. Resistance increases with the number of mutant alleles. The presence of five mutant alleles, the dhfr/dhps "quintuple mutant", including the $d h f r$

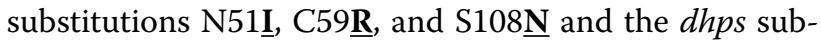
stitutions A437 $\underline{\mathbf{G}}$ and $\mathrm{K} 540 \underline{\mathrm{E}}$, are associated with a very high rate of failure when SP is used for the treatment of uncomplicated falciparum malaria [4]. However, SP remains effective for IPTp, even where the prevalence of the quintuple mutant is high, and thus its use continues to be recommended by the WHO $[5,6]$. The efficacy of SP for IPTp appears to be compromised in the presence

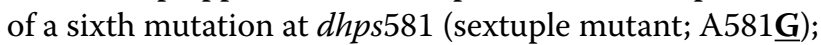
infection with parasites harbouring the sextuple mutant has been associated with increased placental parasitaemia and inflammation and failure of IPTp-SP to improve birth weight [7-9].

Previous studies have reported that the dhps 581 mutation is common in Tanga Region of Tanzania, with up to $57 \%$ of parasites harbouring the sextuple mutant [10]; however, there are limited data from other areas of Tanzania. In light of the possibility that IPTp-SP may provide no benefit to women in areas with a high prevalence of the sextuple mutant, it is critical to define the extent of this mutation. This study aimed to investigate the prevalence of the SP resistance mutations dhps K540E (a surrogate marker for quintuple mutant [4]) and dhps A581G [9] and dhfr I164느 [1] (surrogate markers for sextuple mutant parasites, with A581 $\underline{\mathbf{G}}$ being the more widely reported) in the parasite population in the Lake Zone (Mwanza, Geita, Mara, and Kagera Regions) and Southern Zone (Lindi, Mtwara, and Ruvuma Regions) of Tanzania.

\section{Methods \\ Study area}

From April to June, 2015, a cross-sectional survey was conducted in 14 health facilities (HFs) in seven regions of mainland Tanzania: all four regions in the Lake Zone (Mwanza, Geita, Mara, and Kagera) and all three regions in the Southern Zone (Lindi, Mtwara, and Ruvuma Regions) (Fig. 1). These regions were selected as they had high prevalence of malaria in the 2011/12 Tanzania HIV and Malaria Indicator Survey (THMIS) [11]. In each region, two health facilities were conveniently sampled from facilities with laboratory and where staff had been previously trained on collection of malaria rapid diagnostic tests (RDTs) for other projects to determine whether the dhpsA581G mutation was present in these regions.

\section{Sample size}

It was estimated that a sample size of 100 malaria positive patients from each facility would produce a two-sided 95\% confidence interval of $1.6-11.3 \%$, assuming the true proportion of patients infected with a given mutation was $5 \%$. To account for false positive results that may arise by use of RDTs, the target sample size was inflated by $20 \%$ to approximately 120 samples per facility.

\section{Study population}

All patients age $\geq 6$ months presenting to the outpatient department who were diagnosed with malaria by RDT (Malaria Ag Pf/Pan, SD Bioline ${ }^{\circledR}$ ) or blood smear and no self-reported history of recent exposure to SP or trimethoprim-sulfamethoxazole (within the past 2 months if not pregnant, or during the pregnancy, if pregnant) were consented. In each health facility, consenting eligible patients were consecutively enrolled until the required number of DBS samples (120) were obtained.

\section{Sample collection procedures}

Two blood spots were collected on filter paper (Whatman no. 3, GE Healthcare Life Sciences) at the time the blood sample was collected for malaria testing; if the RDT was negative or the patient did not consent or otherwise did not meet inclusion criteria, the filter paper was destroyed (Fig. 2). Blood spots from consenting individuals were 

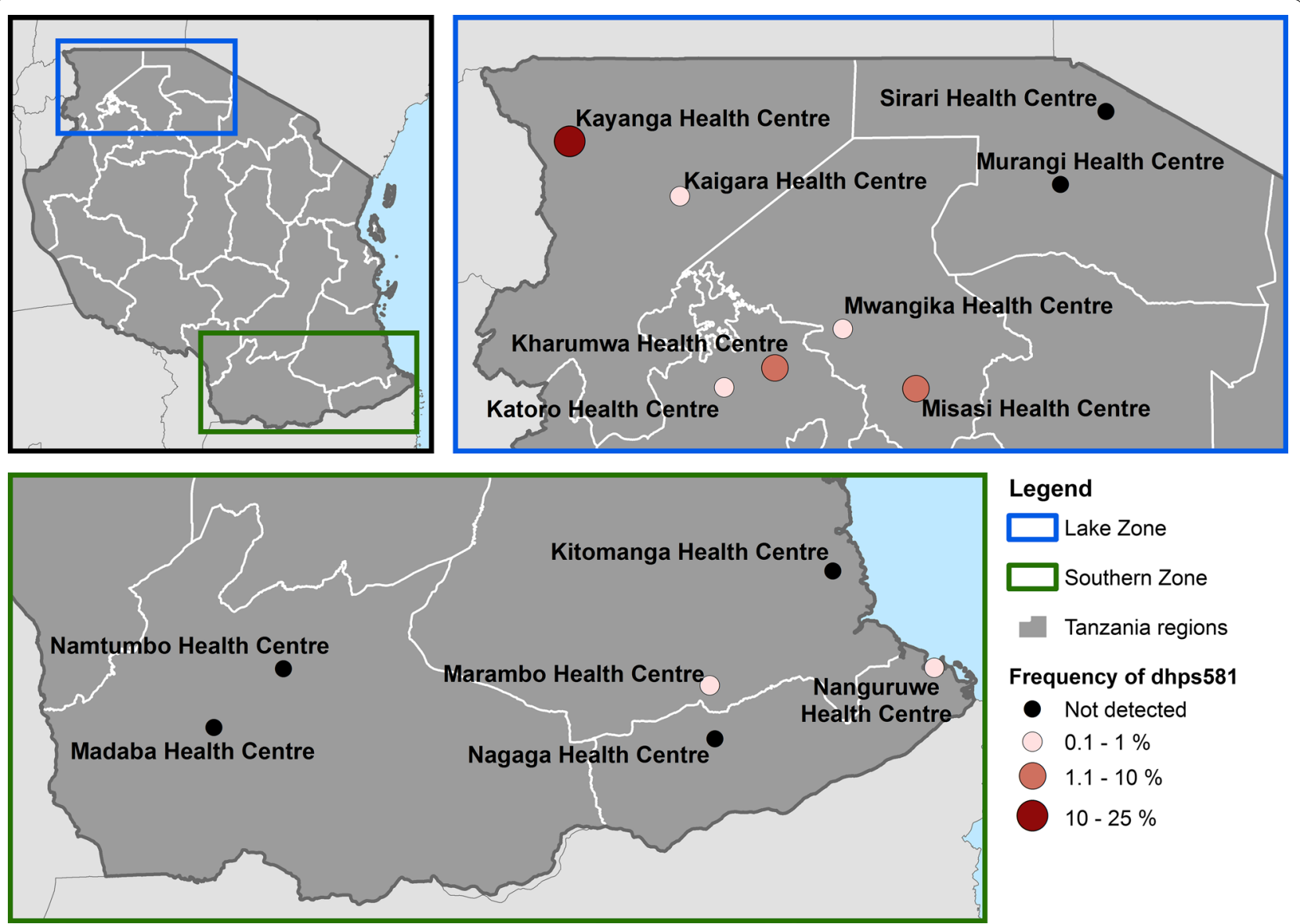

\section{Legend}

$\square$ Lake Zone

Southern Zone

Tanzania regions

\section{Frequency of dhps581}

- Not detected

$0.1-1 \%$

$1.1-10 \%$

$10-25 \%$

Fig. 1 Location of sampled health facilities with frequency of dhpsA581 $\mathbf{G}$ mutations

air dried for 3-4 h on a flat, nonabsorbent surface, then sealed in plastic bags with a desiccant, and stored at room temperature at the study health facility. The samples from all 14 health facilities were packaged in a waterproof container, and shipped to the reference laboratory at the University of North Carolina (UNC) for pooled deep sequencing analyses at three genetic loci associated with sulfadoxine-pyrimethamine (SP) resistance, dhfr164, dhps540, and dhps581 [8, 12-14].

\section{Molecular methods}

\section{Optimizing DNA extraction}

120 Dried Blood Spots (DBS) from the Kharumwa health center were used in a preliminary experiment to determine if DNA from batched DBS could be extracted reliably. Each subject's DBS was punched 3 times. One of these punches was added to a single well of a 96-well plate. The other punches were pooled so that wells would contain either three or ten punches from individuals. The plate was extracted using the Chelex method using overnight incubation at $4{ }^{\circ} \mathrm{C}$ on a shaking platform at
700 RPM (TPM2 shaker, Sarstedt, Nümbrecht, Germany) [15]. After extraction, 3, 9 and $30 \mathrm{ml}$ aliquots of supernatant were removed from the $\mathrm{n}=1, \mathrm{n}=3$, and $\mathrm{n}=10$ extracts, respectively, and combined with aliquots from similarly extracted samples: $1 \times$ : aliquots of all 120 individually extracted samples; $3 \times$ : aliquots of all 40 of the 3-punch extracts; and $10 \times$ aliquots of all 12 of the 10-punch extracts.

Nested PCR amplification was done on each of the three DNA pools for dhps and $d h f r$ using primers shown in Table 1 based on a previous report [16]. PCR conditions were $95^{\circ} \mathrm{C} \times 2 \mathrm{~min}$, followed by 40 rounds of [95 $\left.{ }^{\circ} \mathrm{C} \times 30 \mathrm{~s} \rightarrow 45^{\circ} \mathrm{C} \times 30 \mathrm{~s} \rightarrow 72{ }^{\circ} \mathrm{C} \times 1 \mathrm{~min}\right]$ and a final round of $72{ }^{\circ} \mathrm{C}$ for $10 \mathrm{~m}$. Individual indexed libraries for each gene were made for each DBS pooling condition using the NEB Next Ultra DNA Library Prep Kit (New England Biolabs, Ipswich, USA) and pooled in equimolar fashion. Pooled libraries were sequenced on an Illumina MiSeq using $2 \times 300$ bp reads at the UNC High Throughput Sequencing Facility. Allele frequencies for single nucleotide polymorphisms (SNPs) within the 


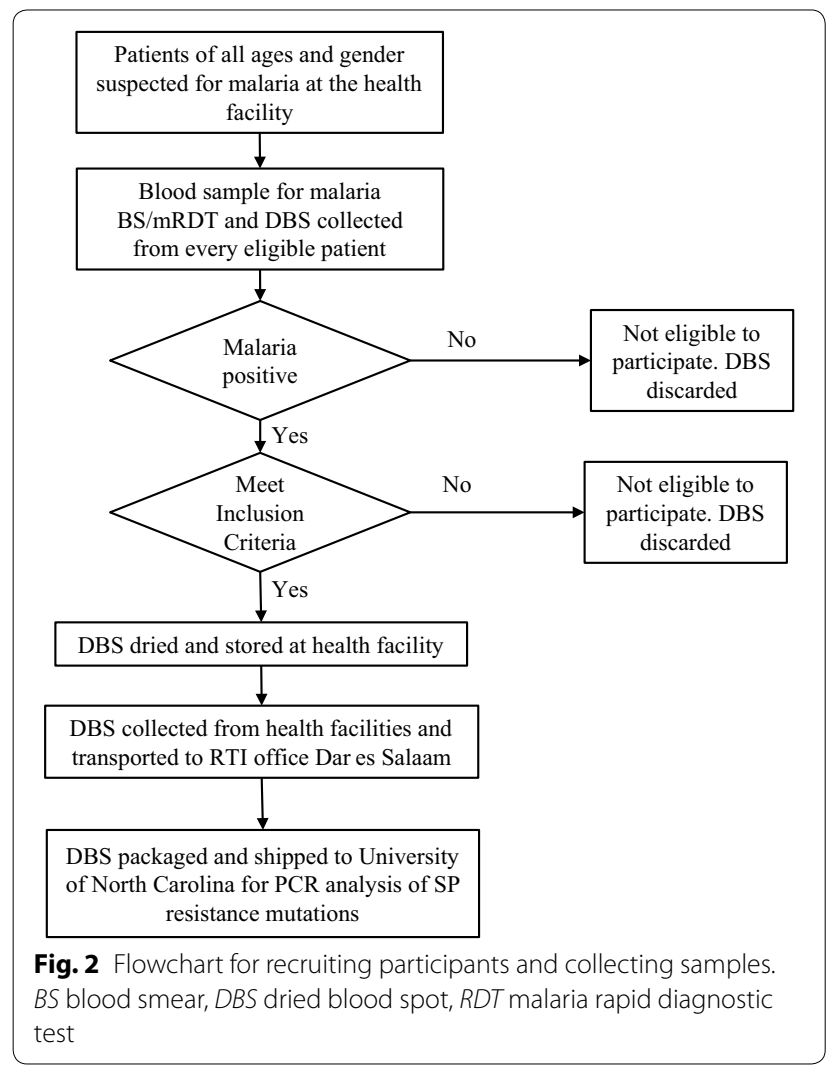

Table 1 Primers

\begin{tabular}{lll}
\hline Primer & Sequence & Descrpition \\
\hline Pfdhfr-F1 & TCCTTTTTATGATGGAACAAG & dhfr outer forward \\
Pfdhfr-R1 & AGTATATACATCGCTAACAGA & dhfr outer reverse \\
Pfdhfr1-F & TGAGGTTTTTAATAACTACACATTTAGA & dhfr inner forward \\
& GGTCT & \\
Pfdhfr1-R & TCGCTAACAGAAATAATTTGATACTCAT & dhfr inner reverse \\
Pfdhps-F1 & AACCTAAACGTGCTGTTCAA & dhps outer forward \\
Pfdhps-R1 & AATTGTGTGATTTGTCCACAA & dhps outer reverse \\
Pfdhps1-F & TGAAATGATAAATGAAGGTGCTAGTGT & dhps inner forward \\
Pfdhps1-R & GTTGTGTATTTATTACAACATTTTGATCA & $d h p s$ inner reverse \\
& TTC & \\
\hline
\end{tabular}

demultiplexed libraries were determined using methods previously described $[17,18]$.

Sequencing reads were aligned using bowtie2 with default settings to the $P$. falciparum 3D7 reference strain and the minor allele frequency (MAF) at all positions across the amplicon was determined using a revised version of our previously described Minor Allele Catcher (MAC) [18]. Bases and reads were excluded from analysis if they had a mapping quality less than or equal to 10 , a read length less than or equal to $200 \mathrm{bp}$, a base quality of less than or equal to 20, a base depth of less than or equal to 5000, if SNPs occurred in the first or last twenty-five bases of a read, or if $30 \%$ or fewer of the reads were on the forward or reverse strand.

\section{Extraction and analysis of DBS from 13 other health centres}

Based on the results of this pilot (Table 2), DNA from all of the remaining samples was extracted in batches of 10 by health centre. The DNA extracts from each of the health centres were then pooled yielding one amplicon pool for each site (13 pools). Library preps and analysis were performed as above. Data from the Kharumwa health centre, 10-punch extract, was re-run a second time to generate the 14-health center dataset.

\section{Results}

A total of 3575 febrile patients were screened, and 1770 were found to be positive for malaria, yielding an overall positivity rate of $49.5 \%$ (Table 2 ). In this pilot work to determine the extraction efficiency of individual versus pooled extraction using the 120 participants from Kharumwa health centre, the proportion of mutant alleles varied only slightly as a result of batching DBS prior to extraction, with $9.4-10.7 \%$ alleles mutated at dhps540, 2.8-3.9\% at dhps581, and none at dhfr164 (Table 3). As these differences in allele frequencies were considered negligible, DBS from all other health centers were extracted in pools of 10 .

A total of 1750 dried blood spot (DBS) samples were collected (117-160 samples per facility) and used in the final study analysis. Deep sequencing resulted in an average of 341,420 reads at dhfr (range 208,769-644,927) and 168,260 reads at dhps (range 49,419-313,645). The library from Kharumwa was re-sequenced during this project. Based upon this repeat sequencing (Table 4) and the 3 sequences in Table 3, the allele frequencies \pm standard deviations for dhps540 and dhps581 mutants were $90.0 \pm 2.1 \%$ and $3.2 \pm 0.7 \%$, respectively. The dhps 540 mutation was common across all 14 sites, with allele frequency ranging from 55 to $98.4 \%$, with higher allele frequency at sites in Lake Zone compared to Southern Zone (Table 4). Frequency of the dhps581 mutation ranged from 0 to $2.4 \%$, with the exception of Kayanga health centre (Kagera Region, Lake Zone) where $24.9 \%$ of sequences were mutated (Fig. 1). The dhfr164 mutation was detected only at Kanyanga health centre (0.6\%).

\section{Discussion}

In a survey of parasites from patients at 14 health facilities in the Lake and Southern Zones of Tanzania, the dhpsK540E mutation was very common [in 10 facilities $(71 \%)$ more than $85 \%$ of alleles at dhpsK540E were mutated, and nowhere were fewer than 55\% mutated], 
Table 2 Malaria testing and positivity rates at selected health centres

\begin{tabular}{|c|c|c|c|c|c|}
\hline Region & District & Health centre & Tested & Positive & Malaria positivity rate (\%) \\
\hline \multicolumn{6}{|l|}{ Lake Zone } \\
\hline Kagera & Karagwe & Kayanga & 331 & 121 & 37 \\
\hline Kagera & Muleba & Kaigara & 292 & 120 & 41 \\
\hline Mara & Musoma & Murangi & 219 & 121 & 55 \\
\hline Mara & Tarime & Sirari & 310 & 122 & 39 \\
\hline Geita & Geita & Katoro & 238 & 122 & 51 \\
\hline Geita & Nyang'hwale & Kharumwa & 230 & 120 & 52 \\
\hline Mwanza & Misungwi & Misasi HC & 187 & 130 & 70 \\
\hline Mwanza & Sengerema & Mwangika & 332 & 121 & 36 \\
\hline Sub-total Lake Zone & & & 2139 & 977 & 46 \\
\hline \multicolumn{6}{|l|}{ Southern Zone } \\
\hline Lindi & Lindi Rural & Kitomanga & 199 & 126 & 63 \\
\hline Lindi & Nachingwea & Marambo & 244 & 129 & 53 \\
\hline Mtwara & Masasi & Nagaga & 222 & 160 & 70 \\
\hline Mtwara & Mtwara & Nanguruwe & 215 & 138 & 64 \\
\hline Ruvuma & Songea & Madaba & 261 & 120 & 46 \\
\hline Ruvuma & Namtumbo & Namtumbo & 295 & 120 & 41 \\
\hline Sub-total Southern Zone & & & 1436 & 793 & 55 \\
\hline Total & & & 3575 & 1770 & 50 \\
\hline
\end{tabular}

Table 3 Distribution of mutants using pools from individually extracted $(1 x)$, 3-punch extracted $(3 x)$ and 10-punch extracted $(10 x)$

\begin{tabular}{lllr}
\hline DNA pool & \multicolumn{4}{l}{ Proportion of mutant alleles } \\
\cline { 2 - 4 } & Dhps540 & Dhps581 & \multicolumn{1}{c}{ Dhfr164 } \\
\hline $1 \times$ & $88.5 \%(339 / 383)$ & $2.8 \%(13 / 464)$ & $0 \%(0 / 319)$ \\
$3 \times$ & $88.6 \%(1014 / 1145)$ & $3.8 \%(52 / 1380)$ & $0.3 \%(2 / 586)$ \\
$10 \times$ & $89.9 \%(563 / 626)$ & $3.3 \%(25 / 765)$ & $0 \%(0 / 401)$ \\
\hline
\end{tabular}

while the dhpsA581G mutation remained rare and focal, with frequency greater than $2.4 \%$ in only one facility, Kayanga Health Facility, where nearly $25 \%$ of alleles carried the dhpsA581G mutation. The quintuple mutation (represented by the dhpsK540E) has been reported widely across Tanzania, with prevalence ranging from 64 to $98 \%$ in one recent report assessing seven regions [10] and 77 to $95 \%$ in another [19]. Previous reports from Tanga Region have found a high prevalence of parasites harbouring the dhps A581 $\underline{\mathbf{G}}$ mutation (44\% in Korogwe [7], $51 \%$ in Muheza [19], and 57\% in Bondo [10]). Although the Lake and Southern Zones of Tanzania are not immediately adjacent to Tanga Region, the absence of the dhpsA581G mutation in the majority of sites highlights that there may be considerable geographic micro-heterogeneity. This is supported by data from Kavishe et al., who similarly found the dhpsA581 $\underline{\mathbf{G}}$ to be present at high proportion in only Tanga and Kagera Regions, though the prevalence of mutants at their sites were higher than the allele frequencies reported here [10]. This is a reassuring finding, suggesting that IPTp-SP retains efficacy in the majority of Tanzania, but highlighting the need for monitoring in multiple geographic sites.

The pooling and sequencing methodology presented here is a cost-effective alternative to individual allele-specific PCR. Using this 2-step pooling methodology, it was possible to perform $90 \%$ fewer DNA extractions and $99 \%$ fewer sequencing runs than if samples from each participant had been sequenced individually, saving a substantial amount of both time and money. The individual extraction and bi-directional Sanger sequencing of 1750 samples would cost on the order of $\$ 19,000$. Here the same data has been compiled for under $\$ 4000$.

Another advantage of the pooling method is that it allows direct calculation of the allele frequency, rather than prevalence. That is, it calculates the percentage of the parasite population bearing the mutation rather than the prevalence of individuals bearing parasites with mutated alleles. From an evolutionary point of view, the allele frequency of a mutation is more important than prevalence of the mutation because it approximates the likelihood that a mosquito will become infected with a mutant parasite after exposure to a given population.

Allele frequency and prevalence can be different when mixed infections are present and when individuals have infections with varying levels of parasitaemia. 
Table 4 Summary of drug resistance allele frequencies

\begin{tabular}{|c|c|c|c|c|c|c|}
\hline \multirow[t]{2}{*}{ Region } & \multirow[t]{2}{*}{ District } & \multirow[t]{2}{*}{ Health centre } & \multirow[t]{2}{*}{ Samples (N) } & \multicolumn{3}{|c|}{ Mutant, \% (number of mutant/total sequences) } \\
\hline & & & & dhps540 & dhps581 & dhfr164 \\
\hline \multicolumn{7}{|l|}{ Lake Zone } \\
\hline Kagera & Karagwe & Kayanga & 120 & $89 \%(154,320 / 173,457)$ & $24.9 \%(46,056 / 185,117)$ & $0.6 \%(3972 / 623,835)$ \\
\hline Kagera & Muleba & Kaigara & 120 & $91.2 \%(270,373 / 296,323)$ & $1 \%(31,77 / 313,669)$ & $0 \%(123 / 286,155)$ \\
\hline Mara & Musoma & Murangi & 117 & $86 \%(160,431 / 186,561)$ & $0 \%(24 / 196,781)$ & $0 \%(20 / 327,806)$ \\
\hline Mara & Tarime & Sirari & 120 & $98.4 \%(262,461 / 266,632)$ & $0 \%(71 / 285,418)$ & $0 \%(17 / 249,213)$ \\
\hline Geita & Geita & Katoro & 122 & $97.2 \%(254,986 / 262,202)$ & $0.2 \%(547 / 280,267)$ & $0 \% 19 / 310,706)$ \\
\hline Geita & Nyang'hwale & Kharumwa & 120 & $92.9 \%(176,055 / 189,500)$ & $2.4 \%(4860 / 201,156)$ & $0 \%(23 / 322,015)$ \\
\hline Mwanza & Misungwi & Misasi & 130 & $92.7 \%(168,879 / 182,105)$ & $2 \%(3773 / 193,348)$ & $0 \%(15 / 348,488)$ \\
\hline Mwanza & Sengerema & Mwangika & 121 & $93.2 \%(177,559 / 190,591)$ & $0.3 \%(692 / 202,087)$ & $0 \%(24 / 386,750)$ \\
\hline \multicolumn{3}{|c|}{ Mean allele frequency Lake Zone* } & 970 & $82.6 \%$ & $3.9 \%$ & $0.1 \%$ \\
\hline \multicolumn{7}{|c|}{ Southern Zone } \\
\hline Lindi & Lindi Rural & Kitomanga & 124 & $94.4 \%(162,087 / 171,760)$ & $0 \%(47 / 177,058)$ & $0 \%(11 / 225,710)$ \\
\hline Lindi & Nachingwea & Marambo & 129 & $77.3 \%(35,351 / 45,753)$ & $0.1 \%(57 / 50,926)$ & $0 \%(41 / 231,375)$ \\
\hline Mtwara & Masasi & Nagaga & 160 & $68.1 \%(107,370 / 157,670)$ & $0 \%(17 / 164,456)$ & $0 \%(46 / 247,292)$ \\
\hline Mtwara & Mtwara & Nanguruwe & 129 & $73.2 \%(104,952 / 143,305)$ & $0.8 \%(1180 / 154,626)$ & $0 \%(17 / 385,082)$ \\
\hline Ruvuma & Songea & Madaba & 120 & $92 \%(71,494 / 77,751)$ & $0 \%(11 / 83,706)$ & $0 \%(13 / 324,890)$ \\
\hline Ruvuma & Namtumbo & Namtumbo & 118 & $55.7 \%(26,134 / 46,901)$ & $0 \%(12 / 52,484)$ & $0 \%(22 / 318,552)$ \\
\hline \multicolumn{3}{|c|}{ Mean allele frequency Southern Zone* } & 780 & $76.8 \%$ & $0.2 \%$ & $0 \%$ \\
\hline
\end{tabular}

* Mean allele frequency for each zone was calculated by averaging the percentage of mutants from each health center

Traditionally, prevalence data have to be transformed by a complex equation to yield predicted allele frequencies [20]. However, in general, the allele frequency approximates the prevalence of major strain in a human population, barring any biases $[16,21]$.

This study has a number of limitations. Samples were collected only from the Lake and Southern Zones, neither of which are immediately adjacent to Tanga region, where the highest prevalence of the dhpsA581G have been reported, thus more studies in those areas are needed to better define the extent of the mutant. With regard to the pooling methodology employed here, while much more cost-effective and time-saving than traditional PCR, it is not possible to trace a parasite strain back to a participant. Also, some haplotypes tend to be amplified better than others, leading to PCR amplification bias. However, newer barcoding methods, such as primer ID [22], compensate for amplification bias and could allow identification of individuals (although DNA would still have to be extracted from individual DBS).

While overall, these data are reassuring with respect to the efficacy of IPTp-SP, there are select areas with a high prevalence of the sextuple mutant, where IPTp-SP may no longer provide a useful benefit against malaria. Spread of this sextuple mutant will threaten the usefulness of SP for IPTp. Given the fact that the quintuple mutant is already found throughout Tanzania, and the sextuple mutant has been found in high prevalence in several sites, continued surveillance in multiple sites, particularly in and around Tanga and Kagera, is warranted to monitor for the spread of the sextuple mutant. The pooling technique presented here provides a highly efficient and cost effective means to screen many samples from multiple sites.

\section{Conclusion}

Although the quintuple mutant was very common at all sampled facilities, dhpsA581G remains geographically restricted, suggesting that IPTp-SP remains effective in most of Tanzania. However, additional surveillance, particularly in and around Tanga and Kagera Region is warranted. This can be achieved efficiently through sequencing pooled samples as described here. Finally, a better understanding of the effect of the dhps 581 mutant on the efficacy of IPTp-SP is needed.

\section{Abbreviations}

BS: blood slide; DBS: dried blood spot on filter paper; IPTp: intermittent preventive treatment in pregnancy; IRS: indoor residual spray; MFP: malaria focal person; MiP: malaria in pregnancy; OPD: outpatient department; RDT: malaria rapid diagnostic test; SP: sulfadoxine-pyrimethamine; WHO: World Health Organization.

\section{Authors' contributions}

JG, JMN, SM, OMM, DSI, RAW designed the study. DSI, SM, OMM, RAW, SL, NK, CK, and LAP oversaw the collection of the samples. SMD, KLT, NJH, JAB, JJJ, and SRM analysed the samples. JG, JMN, and SRM drafted the manuscript which all authors edited and approved. All authors read and approved the final manuscript 


\section{Author details}

${ }^{1}$ RTI International, Dar es Salaam, Tanzania. ${ }^{2}$ National Institute for Medical Research, Tanga, Tanzania. ${ }^{3}$ UNC Gillings School of Global Public Health, Chapel Hill, NC, USA. ${ }^{4}$ National Malaria Control Programme, Dar es Salaam, Tanzania. ${ }^{5}$ US President's Malaria Initiative/United States Agency for International Development, Dar es Salaam, Tanzania. ${ }^{6}$ US President's Malaria Initiative, Malaria Branch, Division of Parasitic Diseases and Malaria, US Centers for Disease Control and Prevention, Dar es Salaam, Tanzania. ${ }^{7}$ Program in Bioinformatics and Integrative Biology, University of Massachusetts Medical School, Worcester, MA, USA. ${ }^{8}$ Malaria Branch, Division of Parasitic Diseases and Malaria, US Centers for Disease Control and Prevention, Atlanta, GA, USA.

\section{Acknowledgements}

We are grateful to the staff of the HCS, NIMR Tanga team (Ezekiel Malecela and August Nyaki), and RTI staff who implemented the study and district malaria focal persons (DMFP) in Lake and Southern Zones for supervising the study.

\section{Competing interests}

The authors declare that they have no competing interests.

\section{Availability of data and materials}

The datasets generated during the current study are available from the corresponding author on reasonable request.

\section{Disclaimer}

The findings and conclusions presented in this manuscript are those of the authors and do not necessarily reflect the official position of the US President's Malaria Initiative, US Agency for International Development, or US Centers for Disease Control and Prevention.

\section{Ethical approval and consent to participate}

The study protocol was approved by the Medical Research Coordinating Committee (MRCC) of the Tanzania National Health Institute for Medical Research (NIMR) [reference NIMR/HQ/R.8a/Nol.IX/1924]. The US Centers for Disease Control and Prevention (CDC) human research protection office reviewed the protocol and granted a non-engaged determination for CDC's role in this project. Informed consent was obtained from all patients/parents or guardians of patients (for minors) participating in the study.

\section{Funding}

This study was made possible through support provided to the Tanzania Vector Control Scale-up Project (Cooperative Agreement 621-A-00-10-00015-00) by the President's Malaria Initiative (PMI) via the US Agency for International Development (USAID) and an inter-agency agreement with the US Centers for Disease Control and Prevention (CDC).

\section{Publisher's Note}

Springer Nature remains neutral with regard to jurisdictional claims in published maps and institutional affiliations.

Received: 15 January 2017 Accepted: 30 May 2017

Published online: 05 June 2017

\section{References}

1. Rogerson SJ, Mwapasa V, Meshnick SR. Malaria in pregnancy: linking immunity and pathogenesis to prevention. Am J Trop Med Hyg. 2007;77:14-22.

2. Desai M, ter Kuile FO, Nosten F, McGready R, Asamoa K, Brabin B, et al. Epidemiology and burden of malaria in pregnancy. Lancet Infect Dis. 2007;7:93-104.

3. Eisele TP, Larsen DA, Anglewicz PA, Keating J, Yukich J, Bennett A, et al. Malaria prevention in pregnancy, birthweight, and neonatal mortality: a meta-analysis of 32 national cross-sectional datasets in Africa. Lancet Infect Dis. 2012;12:942-9.

4. Kublin JG, Dzinjalamala FK, Kamwendo DD, Malkin EM, Cortese JF, Martino $L M$, et al. Molecular markers for failure of sulfadoxine-pyrimethamine and chlorproguanil-dapsone treatment of Plasmodium falciparum malaria. J Infect Dis. 2002;185:380-8.
5. Gutman J, Mwandama D, Wiegand RE, Ali D, Mathanga DP, Skarbinski J. Effectiveness of intermittent preventive treatment with sulfadoxinepyrimethamine during pregnancy on maternal and birth outcomes in Machinga district, Malawi. J Infect Dis. 2013;208:907-16.

6. Kayentao K, Garner P, van Eijk AM, Naidoo I, Roper C, Mulokozi A, et al. Intermittent preventive therapy for malaria during pregnancy using 2 vs 3 or more doses of sulfadoxine-pyrimethamine and risk of low birth weight in Africa: systematic review and meta-analysis. JAMA. 2013;309:594-604.

7. Minja DTR, Schmiegelow C, Mmbando B, Boström S, Oesterholt M, Magistrado $\mathrm{P}$, et al. Plasmodium falciparum mutant haplotype infection during pregnancy associated with reduced birthweight, Tanzania. Emerg Infect Dis. 2013;19:1446-54

8. Harrington WE, Mutabingwa TK, Muehlenbachs A, Sorensen B, Bolla MC, Fried M, et al. Competitive facilitation of drug-resistant Plasmodium falciparum malaria parasites in pregnant women who receive preventive treatment. Proc Natl Acad Sci USA. 2009;106:9027-32.

9. Gutman J, Kalilani L, Taylor S, Zhou Z, Wiegand RE, Thwai KL, et al. The A581 G mutation in the gene encoding Plasmodium falciparum dihydropteroate synthetase reduces the effectiveness of sulfadoxine-pyrimethamine preventive therapy in Malawian pregnant women. J Infect Dis. 2015;211:1997-2005.

10. Kavishe RA, Kaaya RD, Nag S, Krogsgaard C, Notland JG, Kavishe AA, et al. Molecular monitoring of Plasmodium falciparum super-resistance to sulfadoxine-pyrimethamine in Tanzania. Malar J. 2016;15:335.

11. Tanzania Commission for AIDS (TACAIDS), Zanzibar AIDS Commission (ZAC), National Bureau of, Statistics (NBS), Office of the Chief Government Statistician (OCGS), and ICF International. Tanzania HIV/AIDS and Malaria Indicator Survey 2011-12 [Internet]. Dar es Salaam, Tanzania: TACAIDS, ZAC, NBS, OCGS, and ICF International; 2013. http://www.nbs.go.tz/index. php?option=com_content\&view=article\&id=353:2011-12-tanzaniahivaids-and-malaria-indicator-survey-thmis-report\&catid=55:this\&lte $\mathrm{mid}=145$.

12. Plowe CV, Kublin JG, Doumbo OK. P. falciparum dihydrofolate reductase and dihydropteroate synthase mutations: epidemiology and role in clinical resistance to antifolates. Drug Resist Updates. 1998;1:389-96.

13. Gesase S, Gosling RD, Hashim R, Ord R, Naidoo I, Madebe R, et al. High resistance of Plasmodium falciparum to sulphadoxine/pyrimethamine in northern Tanzania and the emergence of dhps resistance mutation at Codon 581. PLoS ONE. 2009;4:e4569

14. Naidoo I, Roper C. Mapping, "partially resistant", "fully resistant", and "super resistant" malaria. Trends Parasitol. 2013;29:505-15.

15. Taylor SM, Antonia AL, Harrington WE, Goheen MM, Mwapasa V, Chaluluka E, et al. Independent lineages of highly sulfadoxine-resistant Plasmodium falciparum haplotypes, eastern Africa. Emerg Infect Dis. 2014;20:1140-8.

16. Taylor SM, Parobek CM, Aragam N, Ngasala BE, Mårtensson A, Meshnick SR, et al. Pooled deep sequencing of Plasmodium falciparum isolates: an efficient and scalable tool to quantify prevailing malaria drug-resistance genotypes. J Infect Dis. 2013;208:1998-2006.

17. Taylor SM, Parobek CM, DeConti DK, Kayentao K, Coulibaly SO, Greenwood BM, et al. Absence of putative artemisinin resistance mutations among Plasmodium falciparum in sub-Saharan Africa: a molecular epidemiologic study. J Infect Dis. 2015;211:680-8.

18. Brazeau NF, Hathaway N, Parobek CM, Lin JT, Bailey JA, Lon C, et al. Longitudinal pooled deep sequencing of the Plasmodium vivax K12 Kelch gene in Cambodia reveals a lack of selection by artemisinin. Am J Trop Med Hyg. 2016;95:1409-12.

19. Baraka V, Ishengoma DS, Fransis F, Minja DTR, Madebe RA, Ngatunga $D$, et al. High-level Plasmodium falciparum sulfadoxine-pyrimethamine resistance with the concomitant occurrence of septuple haplotype in Tanzania. Malar J. 2015;14:439.

20. Hastings IM, Smith TA. MalHaploFreq: a computer programme for estimating malaria haplotype frequencies from blood samples. Malar J. 2008;7:130.

21. Aragam NR, Thayer KM, Nge N, Hoffman I, Martinson F, Kamwendo $\mathrm{D}$, et al. Diversity of T cell epitopes in Plasmodium falciparum circumsporozoite protein likely due to protein-protein interactions. PLOS ONE. 2013;8:e62427.

22. Jabara $C B$, Jones $C D$, Roach J, Anderson JA, Swanstrom R. Accurate sampling and deep sequencing of the HIV-1 protease gene using a primer ID. Proc Natl Acad Sci USA. 2011;108:20166-71. 